\title{
Adelheid v. Saldern \\ Wer ging in die SPD? Zur Analyse der Parteimitgliedschaft in wilhelminischer Zeit
}

Im ersten Moment glaubt man, über die Sozialdemokratische Parteimitgliedschaft in der Zeit des Deutschen Kaiserreiches ausreichend viel zu wissen. Doch anscheinend Bekanntes wird schnell zum Unbekannten. Erstaunt nehmen wir zur Kenntnis, daß es offensichtlich noch keine exakten wissenschaftlichen Untersuchungen über die soziale Zusammensetzung der sozialdemokratischen Mitgliedschaft gibt ${ }^{1}$. Auch die in den letzten Jahren sich im Aufschwung befindende sozio-biographische Methode, bei der es um die Erforschung von historischen Kollektiven geht, machte bislang - vermutlich mangels ausreichender Quellen - vor der sozialdemokratischen Parteimitgliedschaft halt ${ }^{2}$.

In den folgenden Ausführungen wird diese Forschungslücke nicht gefüllt. Stattdessen sollen gegenwärtiges Wissen und verfügbare Interpretationen präsentiert werden, um zu weiteren Fragestellungen und Analysen anzuregen.

Dabei zeigt sich schnell, daß die Entscheidung eines Arbeiters, in die Sozialdemokratische Partei einzutreten, nicht aus einem einzigen Ursachenfaktor erklärt werden

\footnotetext{
${ }^{1}$ So Dieter Fricke, Die deutsche Arbeiterbewegung 1869-1914. Ein Handbuch über ihre Organisation und Tätigkeit im Klassenkampf (Berlin 1976) 264; im folgenden zitiert: Fricke, Arbeiterbewegung. Zur frühen Arbeiterbewegung siehe vor allem Hartmut $Z w a b r$, Die deutsche Arbeiterbewegung im Länder- und Territorienvergleich 1875, in: Geschichte und Gesellschaft 13 (1987) $448-507$.

${ }^{2}$ Zur sozio-biographischen Methode siehe Wilbelm Heinz Scbröder (Hrsg.), Lebenslauf und Gesellschaft. Zum Einsatz von kollektiven Biographien in der historischen Sozialforschung (Stuttgart 1985) passim; im folgenden zitiert: Scbröder, Lebenslauf. Schröder definiert die kollektive Biographie als eine „theoretisch und methodisch reflektierte, empirische, besonders auch quantitativ gestützte Erforschung eines historischen Personenkollektivs in seinem jeweiligen gesellschaftlichen Kontext anhand einer vergleichenden Analyse der individuellen Lebensläufe der Kollektivmitglieder“. Ebda., 8. Die sozio-biographische Methode wurde bislang auf sozialdemokratische Führer angewandt. Dazu siehe z. B. Eberhard Kolb, Zur Sozialbiographie einer Führungsgruppe der SPD am Anfang der Weimarer Republik: Die Mitglieder des „Zentralrats“ 1918/19, in: Herkunft und Mandat. Beitrāge zur Führungsproblematik in der Arbeiterbewegung (Schriftenreihe der Otto-Brenner-Stiftung 5, Frankfurt/Köln 1976); Wilbelm Heinz Scbröder, Die Sozialstruktur der sozialdemokratischen Reichstagskandidaten 1898 bis 1912, in: Herkunft und Mandat. Beiträge zur Führungsproblematik in der Arbeiterbewegung (Frankfurt/Köln 1976); Christel Wickert, Frauen im Parlament: Lebensläufe sozialdemokratischer Parlamentarierinnen in der Weimarer Republik, in: Scbröder (Hrsg.), Lebenslauf, 210-241.
} 
kann. Die Vorstellung, daß vor allem die ökonomische Klassenlage und die schlechten Lebensverhältnisse zum Anschluß an die SPD geführt hätten, wäre zwar nicht falsch ${ }^{3}$, aber unzureichend und z.T. irreführend. Zwar erreichte die Zahl der Mitglieder der Sozialdemokratischen Partei Deutschlands im Jahre 1913/14 die Eine-MillionGrenze , aber insgesamt gesehen organisierten sich doch „nur wenige“ Arbeiter in der Partei (der Anteil lag unter 10\%), und dies, obwohl die meisten Arbeiter an ihre objektive Klassenlage fest gebunden blieben. Es muß also auch noch nach anderen Ursachenfaktoren gesucht werden. Nicht zufällig kommt bei dieser Suche der soziokulturelle Bereich (stärker als früher) ins Blickfeld. Es gilt, auch die Wirkungsweise der (politischen) Sozialisation, des Sozialmilieus, der (Berufs-)ausbildung etc. zu berücksichtigen $^{6}$; ebenso müssen Lebenserfahrungen, Vergangenheits- und Gegenwartsdeutungen sowie Zukunftserwartungen in die Analyse mit einbezogen werden ${ }^{7}$. Ein solcher multiperspektivischer analytischer Zugriff geht freilich über eine rein quantitative Untersuchung der sozialen Struktur der Mitgliedschaft hinaus; qualitative Aspekte kommen hinzu.

Fraglos sind Analysen von Parteimitgliedschaften nützlich, gleichwohl sollten diese in ihrem Aussagewert nicht überschätzt werden. Dies gilt auch für die wilhelminische SPD. Beispielsweise nach der sozialen Basis des Reformismus oder des Radikalismus

${ }^{3}$ So gibt es selbstverständlich Zusammenhänge zwischen der regionalen Sozialstruktur und der Entwicklung der sozialdemokratischen Mitgliedschaft (vgl. hierzu G. A. Ritter in diesem Band).

${ }^{4}$ Fricke, Arbeiterbewegung, 245. Im Jahre 1905/06 waren es erst 384327 Mitglieder. Genauere Angaben über die zahlenmāßlige Entwicklung der Gesamtpartei liegen erst seit 1905/06 vor, abgesehen von einzelnen regionalen und örtlichen Parteiorganisationen. Näheres dazu siehe ebd., $244 \mathrm{f}$

${ }^{5}$ So belief sich der Anteil der Parteimitglieder an der gesamten Arbeiterschaft in Augsburg 1901 auf $0,8 \%, 1905$ auf $1,8 \%, 1908$ auf $3,9 \%, 1911$ auf $5,5 \%$. Ilse Fischer, Industrialisierung, sozialer Konflikt und politische Willensbildung in der Stadtgemeinde. Ein Beitrag zur Sozialgeschichte Augsburgs 1840-1914 (Schriftenreihe des Stadtarchivs Augsburg 24, Augsburg 1977) 334; im folgenden zitiert: Fischer, Augsburg. - In Göppingen gehörten weniger als 6\% der örtlichen Arbeiterschaft der SPD an. Dieter Wuerth, Radikalismus und Reformismus in der sozialdemokratischen Arbeiterbewegung Göppingens, 1910-1919 (Göppingen 1978) 54; im folgenden zitiert: Wuerth, Göppingen. - In Frankfurt betrug der Anteil (1906) 4,7\%, in Nürnberg 21,1\%.

${ }^{6}$ In diesem Zusammenhang sollte auf die "Dialektik von objektiven und einverleibten Strukturen" hingewiesen werden. Nach Bourdieu sind diese einverleibten Strukturen zwar nicht linear aus der Klassenlage ableitbar, aber auch nicht ohne Analyse der Klassenlage erklärbar: Die für einen spezifischen Typus von Umgebung konstitutiven Strukturen (etwa die eine Klasse charakterisierenden materiellen Existenzbedingungen) erzeugen sog. Habitusformen. Solche „strukturierten Strukturen" seien ihrerseits geeignet, „als strukturierende Strukturen zu wirken“, indem sie nämlich Praxisformen erzeugen und strukturieren und so Geschichte machen. Die Stärke der Überlegungen Bourdieus liegt im Aufzeigen der Bedingungen für die Entstehung eines relativ konstanten Habitus, während Verānderungen des Habitus nicht erklärt werden. Gerade aber der Bruch mit dem regelhaft wirkenden Habitus ist für die Erklärung jenes Schrittes, der schließlich zum Pateieintritt führte, wichtig. Vgl. Pierre Bourdieu, Entwurf einer Theorie der Praxis auf der ethnologischen Grundlage der kabylischen Gesellschaft (Frankfurt 1979) 162, 164 f., 169.

${ }^{\top}$ Hinsichtlich der Bedeutung der Erfahrungen für Handlungsweisen und Klassenbildungsprozesse vgl. Eduard P. Thompson, The Making of the English Working Class (Harmondsworth 1963) vor allem 9-14, 189-212. 
zu suchen, dürfte vergebliches Bemühen $\operatorname{sein}^{8}$. So konnte zwar festgestellt werden, daß jene Ortsvereine, in denen die Metaller quantitativ überwogen, stärker als andere Ortsvereine zu einer Linksorientierung neigten. Dies trifft jedoch beispielsweise für die Bielefelder SPD, in der ebenfalls die Metaller dominierten, nicht $\mathrm{zu}^{9}$. Allerdings war die soziale Basis bei der Durchsetzung einer bestimmten Parteilinie keineswegs bedeutungslos, vor allem dann nicht, wenn Fragen nach Erfahrungshorizonten und subjektiven Realitātsdeutungen eingeschlossen werden. Dabei müssen auch die Ungleichzeitigkeiten hinsichtlich der regionalen und branchenbezogenen Entwicklungen berücksichtigt werden ${ }^{10}$. Diese bestanden - ungeachtet der Entwicklung der SPD zur Massenpartei seit 1890/1900 - weiter fort.

Die folgenden Ausführungen konzentrieren sich auf zwei Punkte: Zum einen geht es um eine klassen-/schichten- und berufsspezifische Untersuchung der sozialdemokratischen Mitgliedschaft. Zum anderen wird versucht, über den Entscheidungsprozeß, der schließlich zum Eintritt in die Partei führte, einige Aussagen bezüglich der sozio-kulturellen Dispositionen zu machen. Hierbei kommt es notgedrungen auch zu bloßen Vermutungen und mehr oder weniger stichhaltigen Andeutungen. Außerdem müssen Hilfskonstruktionen angewandt werden, indem auch aus der Analyse jener Personengruppen, die nicht in die SPD eintraten, Rückschlüsse für unsere Fragestellung gezogen werden.

\footnotetext{
${ }^{8}$ Wichtig ist freilich, den Reformismus allgemein als eine Folge einer bestimmten Wahrnehmungsweise gesellschaftlicher Realitāt zu begreifen; dazu: Adelheid 2 : Saldern, Auf dem Weg zum Arbeiter-Reformismus. Parteialltag in sozialdemokratischer Provinz. Göttingen 1870-1920 (Frankfurt 1984), vor allem die Einleitung; im folgenden zitiert: $v$ : Saldern, Arbeiter-Reformismus. W. L. Guttsman, The German Social Democratic Party 1875-1933 (London 1981) 160 f.; im folgenden zitiert: Guttsman, Social Democratic Party. Erinnert sei in diesem Zusammenhang an das stark kritisierte Buch von Karl-Heinz Roth, Die „andere“ Arbeiterbewegung und die Entwicklung kapitalistischer Repression von 1880 bis zur Gegenwart. Ein Beitrag zum Neuverstāndnis der Klassengeschichte in Deutschland (München 1974).

9 Karl Ditt, Industrialisierung, Arbeiterschaft und Arbeiterbewegung in Bielefeld 1850-1914 (Untersuchungen zur Wirtschafts-, Sozial- und Technikgeschichte 4, Dortmund 1982) 251; im folgenden zitiert: Ditt, Bielefeld.

${ }^{10}$ Der Begriff der Ungleichzeitigkeit geht auf die Überlegungen von Ernst Bloch zurück, wobei dieser allerdings weniger an regionale Ungleichzeitigkeiten, sondern mehr an unterschiedliche Mentalităten einzelner sozialer Gruppen dachte - unterschiedliche Mentalitäten, die im Grunde verschiedenen Zeitepochen zugehörten. Fruchtbar an diesem Gedanken ist die Erkenntnis, daß auch Regionen von Ungleichzeitigkeiten bestimmt werden, die aber nicht nebeneinander, $d . h$. nicht getrennt voneinander existieren, sondern sich überlagern - in mehrdimensionaler Verflechtung miteinander, aber auch gegeneinander stehend. Dazu vgl. Ernst Bloch, Erbschaft dieser Zeit (Frankfurt 1962) bes. 104 ff.; siehe auch die exemplarische Studie von Gerd Zang (Hrsg.), Provinzialisierung einer Region. Regionale Unterentwicklung und liberale Politik in der Stadt und im Kreis Konstanz im 19. Jahrhundert. Untersuchungen zur Entstehung der bürgerlichen Gesellschaft in der Provinz (Frankfurt 1978); im folgenden zitiert: Zang, Provinzialisierung.
} 


\section{Soziale und berufliche Herkunft der sozialdemokratischen Parteimitglieder}

\section{Klassen- und schichtenbezogene Merkmale}

Einst zweifelten Zeitgenossen - so Robert Blank - daran, daß die SPD überhaupt eine Klassenpartei, d.h. eine Arbeiterpartei war ${ }^{11}$. Diese Auffassung wurde widerlegt ${ }^{12}$. Gemessen an dem überwiegenden Anteil gelernter und ungelemter Arbeiter in der Mitgliedschaft (in der Regel 80-90\%) war die wilhelminische SPD eine Arbeiterpartei $^{13}$.

Sowohl in den Parteizentren ${ }^{14}$ als auch in den Parteiprovinzen überwog bei weitem die „breite Schicht der mittleren Arbeiter"15. Zu ihr gehörten hauptsächlich gelernte

${ }^{11}$ So veröffentlichte im Jahre 1905 der Soziologe R. Blank seine Untersuchungen über die Wählerschaft der SPD. Er behauptete, daß der Anteil der in der Großindustrie beschäftigten Arbeiter „numerisch kaum stärker" zu Buche schlüge als der Anteil der bürgerlichen Elemente. Deshalb sei die SPD nach ihrer sozialen Zusammensetzung keine Klassenpartei. Robert Blank, Die soziale Zusammensetzung der sozialdemokratischen Wählerschaft Deutschlands, in: Archiv für Sozialwissenschaft und Sozialpolitik NF XX (1905) 523, 539. Innerhalb der SPD wurde damals ebenfalls die Relevanz klassenfremder Elemente für die Partei diskutiert. Dazu siehe einführend vom Standpunkt der heutigen DDR: Fricke, Arbeiterbewegung, $253 \mathrm{ff}$. Die Kernfrage lautete, ob die Existenz nichtproletarischer Schichten in der SPD sich auch auf Ziele und Strategien der Partei auswirken würde. Diese Frage wurde aber mehr unter qualitativem (und weniger unter quantitativem) Aspekt diskutiert.

${ }^{12}$ Dazu siehe schon Gerbard A. Ritter, Die Arbeiterbewegung im wilhelminischen Deutschland (Berlin ${ }^{2} 1963$; Erstauflage 1959) $77 \mathrm{f}$.

${ }^{13}$ Vgl. Fricke, Arbeiterbewegung, 265. In Göttingen machte der Prozentsatz der proletarischen Mitglieder 95,7 aus. Adelbeid v. Saldern, Vom Einwohner zum Bürger. Zur Emanzipation der städtischen Unterschicht Göttingens 1890-1920. Eine sozial- und kommunalhistorische Untersuchung (Schriften zur Wirtschafts- und Sozialgeschichte 21, Berlin 1973) 114; im folgenden zitiert v. Saldern, Einwohner. - In Düsseldorf waren 98\% der gesamten Mitgliedschaft 1906 der Arbeiterklasse zugehörig, in Dortmund waren es $85 \%$. Mary Nolan, Social Democracy and Society. Working-Class Radicalism in Düsseldorf 1890-1920 (Cambridge University Press 1981) 102, im folgenden zitiert: Nolan, Düsseldorf. In München machte der Anteil der Lohnarbeiterschaft (1906) „nur“ $80 \%$ aus. Robert Michels, Die deutsche Sozialdemokratie. Mitgliedschaft und soziale Zusammensetzung, in: Archiv für Sozialwissenschaft und Sozialpolitik NF XXIII (1906) 509, im folgenden zitiert: Michels, Sozialdemokratie.

${ }^{14}$ Für viele Fragen der Parteigeschichte ist eine Differenzierung zwischen Parteizentren und Parteiprovinzen angebracht. Dabei zeichnet sich die Entwicklung der deutschen Sozialdemokratie dadurch aus, daß sie sich nicht von einem einzigen Zentrum aus entwickelte, sondern daß von Anfang an mehrere regionale Parteizentren vorhanden waren. Nach vorläufigen Recherchen haben sich die Unterschiede zwischen Parteizentren und Parteiprovinzen in wilhelminischer Zeit eher vergrößert als verringert, $d$. h. die Parteiprovinzen gerieten stärker in eine parteiorganisatorische und parteikulturelle Abhāngigkeit von den (regionalen) Parteizentren. Dazu siehe neuerdings Adelheid v. Saldern, Parteizentren und Parteiprovinzen. Zu Fragen der regionalen Differenzierung und Hierarchisierung der wilhelminischen SPD (Vortragsmanuskript, deutsch-polnische Konferenz in Poznań, Oktober 1987; erscheint in polnisch); im folgenden zitiert: $v$. Saldern, Parteizentren. Allerdings gibt es auch Städte und Regionen, die sich weder dem einen noch dem anderen Typ zuordnen lassen, so z. B. die vielen industrialisierten ländlichen Gemeinden im Königreich Sachsen (vgl. G. A. Ritter in diesem Band).

is Fricke, Arbeiterbewegung, 271; Wuerth, Göppingen, $59 \mathrm{f}$. 
Arbeiter (in Bielefeld lag der Prozentsatz (1904) bei 61,2 v. H.) ${ }^{16}$, von denen viele handwerkliche Berufe ausübten. Allerdings stieg mit der Zeit der Anteil der gelernten Industriearbeiter (Facharbeiter).

Die sog. Arbeiteraristokratie - also nicht nur der gelernte, sondern auch der in seinem Beruf hochqualifizierte Arbeiter in relativ guter Position und mit verhältnismäßig hohem Verdienst - machte innerhalb der Arbeiterklasse nur eine relativ kleine soziale Gruppe aus, so daß es nicht verwundert, wenn diese auch in der SPD quantitativ gesehen wenig vertreten war ${ }^{17}$. Überdies setzte ein Teil der obersten Arbeiterschicht auf den individuellen Aufstieg und hielt sich deshalb bewußt von der Partei fern. In Göttingen beispielsweise bestand die Arbeiteraristokratie vor allem aus Feinmechanikem, diese waren aber nur vereinzelt in der Partei zu finden ${ }^{18}$.

Ungelemte Arbeiter blieben in der Partei vor 1914 unterrepräsentiert ${ }^{19}$. Man kann davon ausgehen, daß die ungelernten - zusammen mit den angelemten - Arbeitern (soweit diese als Berufsangabe den Begriff ,Arbeiter“ verwendeten) zwischen 10 und $25 \%$ der sozialdemokratischen Mitgliedschaft ausmachten. Bemerkenswert ist, daß in Parteiprovinzen die ungelernten Arbeiter hāufig einen höheren Anteil an der Mitgliedschaft stellten als in den Parteizentren. So waren in einem regionalen Parteizentrum wie Nürnberg 1894 nur 9,3\% der Mitglieder als ungelernte Arbeiter ausgewiesen, während der Prozentsatz in der Parteiprovinz Göttingen 1906/07 bei $25 \% \operatorname{lag}^{20}$.

\footnotetext{
${ }^{16}$ Ditt, Bielefeld, 250.

17 Vgl. Fricke, Arbeiterbewegung, 271.

${ }^{18}$ Von der Gesamtzahl der Beschäftigten in der Metallbranche Göttingens waren nur 1,2\% in der SPD organisiert, und nur 2 der Sozialdemokraten bezeichneten sich als Mechaniker; $\imath$ : Saldern, Einwohner, $117 \mathrm{f}$. Es fehlen noch ausreichende qualitative Längsschnittanalysen über Denkweisen und Verhalten der Arbeiteraristokratie. In diesem Zusammenhang müßten auch die Wohnmilieus beachtet werden. Für Hamburg stellt Kutz-Bauer fest, daß die sozialdemokratische Wählerschaft vor allem in den Arbeiterquartieren wohnte, während die aktiven Sozialdemokraten eher in den sozial gernischten Quartieren zu finden waren, „wo eine bescheidene Kleinbürgerlichkeit dominiert und Gewerbe und Kleingewerbe seine Standorte hat". Helga Kutz-Bauer, Arbeiterschaft, Arbeiterbewegung und bürgerlicher Staat in der Zeit der großen Depression (Bonn 1988) 422. Für die zwanziger Jahre siehe unter solcher Fragestellung Adelheid v. Saldern, Die Neubausiedlungen der zwanziger Jahre, in: Ulfert Herly'n, Adelbeid $t$ : Saldern, Wulf Tessin (Hrsg.), Neubausiedlungen der zwanziger und sechziger Jahre. Ein historisch-soziologischer Vergleich (Frankfurt/New York 1987). In den meisten Untersuchungen wird allerdings nur zwischen gelernten Arbeitern (welche über die Berufsangabe zu erschließen sind) und ungelernten Arbeitern, die sich dann auch als Arbeiter bezeichneten, unterschieden, nicht aber zwischen der breiten Masse der gelemten Arbeiter und den Spitzenfacharbeitern. Ebenso schwierig ist es, die angelernten Arbeiter als eigene Gruppe herauszudestillieren.

${ }_{19}$ Fricke, Arbeiterbewegung, $271 \mathrm{f}$. Es muß auch bedacht werden, daß es $\mathrm{zwar}$ sinnvoll ist, zwischen gelernten und ungelernten Arbeitern zu unterscheiden, daß damit aber wenig über die spezifische Position im Betrieb bzw. über den sich verāndemden Arbeitsplatz ausgesagt wird. Ebda., $272 \mathrm{f}$.

${ }^{20}$ Dieter Rossmeisl, Arbeiterschaft und Sozialdemokratie in Nürnberg 1890-1914 (Schriftenreihe des Stadtarchivs Nürnberg, Nürnberg 1977) 207 f.; im folgenden zitiert: Rossmeisl, Nürnberg. Unter den 191 Personen, die als ungelemte Arbeiter ausgewiesen wurden, befand sich eine Gruppe
} 
Die relativ größere Repräsentanz ungelemter (bzw. angelemter) Arbeiter in den Parteiprovinzen schwächte jedoch eher die Position der Parteiprovinzen gegenüber den Parteizentren, denn in der Gesamtpartei waren es ja mehr die gelernten Arbeiter, die den Ton angaben und deren Interessen innerhalb der Organisation am meisten Berücksichtigung fanden.

Die aus dem Produktionsprozeß Herausgeschleuderten, beispielsweise die sog. "Wanderarmen“ oder die Gelegenheitsarbeiter waren bezeichnenderweise in der Partei von vornherein gar nicht vertreten. Vielleicht wird an dieser Stelle deutlich, daß die soziale Basis der SPD in nur eingeschrānktem Ausmaße mit der „Kultur der Armut“ verbunden war, nämlich nur insoweit, als (ungelernte) Arbeiter überhaupt am Produktionsprozeß in relativ geregelter Weise teilhatten (s. auch unten) ${ }^{21}$.

Der Anteil der nicht-proletarischen Mitglieder der wilhelminischen SPD lag zwischen 5-20\%. Die meisten nicht-proletarischen Mitglieder kann man wohl zum Kleinbürgertum rechnen (selbstāndige Handwerker, Einzelhändler, kleine Kaufleute, Hausierer, Gastwirte, kleine Angestellte). Den höchsten Anteil an Kleinbürgern hatte die Münchner SPD mit 21,6\% aufzuweisen (1906) ${ }^{22}$. Es war dies wohl die Folge eines langsameren Übergangs zur Industrialisierung dieser Stadt als es sonst üblich war - bei gleichzeitig stark ausgeprägter gewerblicher Mischstruktur. - Auch in diesem Punkt gab es übrigens zwischen Parteiprovinzen und Parteizentren gewisse Unterschiede: Es scheint, als ob das Kleinbürgertum in den Parteiprovinzen prozentual nicht so stark repräsentiert war wie in den Parteizentren ${ }^{23}$.

Allerdings muß man bei diesen Zahlen beachten, daß nicht selten sich sozialdemokratische Kleinbürger früher in lohnabhängiger Stellung befunden hatten, bevor sie sich selbstāndig machten. (Hāufig wurden sie auf Grund ihrer Parteitätigkeit zu die-

von 94 Personen, die mit der Bezeichnung „Fabrikarbeiter und Tagelöhner“ gesondert aufgeführt wurden. Es sind damit wohl ungelernte Hilfsarbeiter gemeint, während es sich bei dem Rest um angelemte Arbeiter gehandelt haben dürfte. - In Berlin wies die Statistik über die berufliche Zusammensetzung der Mitgliedschaft im Jahre 1906 14,9\% der Mitglieder (= 7903) als „Arbeiter" aus. Dabei dürfte es sich ebenfalls um ungelernte Arbeiter gehandelt haben. Fricke, Arbeiterbewegung, 271. In Hamburg betrug der Anteil der als (ungelernte) Arbeiter ausgewiesenen Personen 1898 23\% (2655) und 1903 20,6\% (3394) der Mitglieder; ebda., 256. Aus Göppingen wird berichtet, daß dort ungelernte Arbeiter relativ selten in der Partei organisiert waren, ohne daß genaue Zahlen genannt werden. Wuerth, Göppingen, 59. In Bielefeld betrug der Prozentsatz der un- bzw. angelernten Arbeiter in der Partei 1904 32,8\% (882 Personen); Ditt, Bielefeld, 250.

${ }^{21}$ Dazu neuerdings einführend Michael Grüttner, Die Kultur der Armut, in: Soziale Bewegungen (Jb. 3, Frankfurt/New York 1987) 12 ff.; im folgenden zitiert: Grüttner, Armut; zu den Wanderarmen siehe vorläufig v. Saldern, Einwohner, $218 \mathrm{f}$.

${ }^{22}$ Michels, Sozialdemokratie, 509. Auch hier erfolgte die Zurechnung nicht nach strikten Festlegungen. Dementsprechend sind alle Zahlen mit Vorbehalt zu lesen.

${ }_{23} v$. Saldern, Einwohner, 114. Der Anteil der Kleinbürger an der Parteimitgliedschaft lag in Marburg mit 4,4\% und in Frankfurt mit 5,7\% ähnlich hoch wie in Göttingen. Michels, Sozialdemokratie, 509. Fricke geht davon aus, daß nicht wenige Sozialdemokraten vor ihrem Anschluß an die Partei ebenfalls kleinbürgerlichen Schichten angehört hätten; diese seien erst infolge der zunehmenden Konzentration und Zentralisation des Kapitals zu Proletariem geworden. Fricke, Arbeiterbewegung, 249 . 
sem Schritt mehr oder weniger gezwungen ${ }^{24}$. Im übrigen weiß man, daß viele „Selbstāndige“ - vor allem Schneider und Schuhmacher - ebenfalls eine quasi-proletarische Existenz fristeten (Einmannbetriebe).)

Zum Kleinbürgertum zählten auch die Gastwirte. Diese spielten bekanntlich in der Partei eine wichtige Rolle insofern, als die Partei über viele Jahre hinweg geeignete Gastwirtschaftsräume benötigte, um ihre Versammlungen abhalten zu können. In den Parteizentren waren Gastwirte in der SPD relativ stark vertreten - im Unterschied zu einer Parteiprovinz wie Göttingen ${ }^{25}$. Dieser Unterschied resultiert aus der Tatsache, daß im Falle Göttingen die Gastwirte mehr verdienten, wenn sie sich den Militärpersonen und der Studentenschaft öffneten - und das schloß in der Praxis eine sozialdemokratische Kundschaft eben aus.

Das mittlere und gehobene Bürgertum war in der SPD nur marginal vertreten. Es handelte sich dabei vor allem um Akademiker und sonstige Intellektuelle. Diese waren vorwiegend in den Parteizentren zu finden, kaum jedoch in den Parteiprovinzen, erst recht nicht in kleinen Universitātsstādten wie Göttingen und Tübingen. Dies erklärt sich vor allem daraus, daß dort die Intelligenz mit den weitgehend illiberalen (und antisozialdemokratisch gesinnten) Universitāten verbunden blieb, während das großstädtische Milieu auch andere Typen von Intellektuellen (Literaten, Redakteure etc.) hervorbrachte, die z.T. der SPD gegenüber recht aufgeschlossen waren. Obwohl auch in den Parteizentren die Akademiker quantitativ gesehen nur eine geringe Zahl ausmachten, war der qualitative Einfluß auf die Partei relativ groß und darüberhinaus auch noch in der Partei sehr umstritten (vgl. die sog. „Akademikerfrage") ${ }^{26}$.

${ }^{24}$ Dies gilt auch für den Vorsitzenden der Göttinger SPD, Wilhelm Stegen. Vgl. v. Saldern, Arbeiter-Reformismus, 299. Für Augsburg vgl. Fischer, Augsburg, 334. In Düsseldorf waren zwischen 1896 und 1908 nur 33 kleine Händler, Angestellte, Beamte, Gastwirte usw. in die Partei eingetreten. (1908 waren es dann gleich auf einen Schlag 49 Personen.) Nolan, Düsseldorf, 102. In Bielefeld machten die Kaufleute und Wirte in der SPD (1904) 5,6\% aus. Hinzu kamen noch $0,5 \%$ Angestellte. Ditt, Bielefeld, 250 .

${ }^{25}$ In Leipzig betrug der Anteil der Gastwirte an der Parteimitgliedschaft 1904 3,4\% 63 Personen). Michels, Sozialdemokratie, 514. In Göttingen waren 1908 nur 3 Gastwirte in der Partei organisiert; 2 : Saldern, Einwohner, 115.

${ }_{26}$ Akademiker und Intellektuelle, welche in der SPD in Bremen, Berlin, München und Leipzig eine so große Rolle spielten, waren beispielsweise in Düsseldorf so gut wie gar nicht in der Partei vertreten; Nolan, Düsseldorf, 102. - In der SPD wurde damals intensiv über die Funktion der Intellektuellen in der Partei diskutiert. Die Auseinandersetzung (die sog. „Akademikerfrage“) stand bekanntlich im Kontext des Konfliktes um den parteipolitischen Kurs. Es handelte sich also nicht um einen Antiintellektualismus, wenngleich Vorbehalte durchaus vorhanden waren. Vgl. dazu Benno Fischer, Sozialdemokratie und Intelligenz in der Weimarer Republik, in: Richard Saage (Hrsg.), Solidargemeinschaft und Klassenkampf. Politische Konzeptionen der Sozialdemokratie zwischen den Weltkriegen (Frankfurt 1986) 223; im folgenden zitiert: Fischer, Intelligenz. Auch Boll sieht im Faktor ,intellektuelles Milieu“ einen wichtigen Unterschied zwischen Braunschweig und Hannover. Wāhrend in Braunschweig eine recht große intellektuelle Diskussionsfreudigkeit geherrscht habe, war die Hannoversche Partei mehr eine auf pragmatisches Handeln ausgerichtete „Gewerkschaftspartei“ (mündl. Hinweis v. Autor); vgl. auch seine Studie: Friedhelm Boll, Massenbewegungen in Niedersachsen 1906-1920. Eine sozialgeschichtliche Untersuchung zu den unterschiedlichen Entwicklungstypen Braunschweig und Hannover (Bonn 1981) bes. 115; im folgenden zitiert: Boll, Massenbewegungen. 
Eine spezifische Gruppe der Intelligenz stellten die jüdischen Intellektuellen dar. Ihre kulturelle Schwebelage und ihr nicht selten sehr scharf-kritischer Blick auf die zeitspezifischen Ausprägungen der militaristisch-kapitalistischen Gesellschaft führten nicht selten zu systemoppositionellen, also zu sozialdemokratischen bzw. sozialistischen Ideen. Hieraus erklärt sich, daß ,geistes- und kulturgeschichtliche Elemente des jüdischen Denkens gleichsam immanente Bestandteile der Sozialismus-Diskussion waren und sind und von dieser Seite aus auch auf das gesellschaftliche und politische Denken in Deutschland seit langem einwirken" ${ }^{\text {"27. }}$.

\section{Berufsspezifische Merkmale}

In berufsbezogenen Statistiken über die Parteimitglieder ${ }^{28}$ dominierten meist die Branchen Metall, Bau und Holz. Dies gilt z. B. auch für Düsseldorf. Nolan hat in ihrer Untersuchung über die Düsseldorfer Arbeiterbewegung diese Tatsache zu erklären versucht: Es habe sich hierbei um gelernte Leute gehandelt, die eine vorteilhafte Position auf dem Arbeitsmarkt eingenommen und über relativ qualifizierte Arbeitsplätze verfügt hătten. Sie betont ferner die gemeinsame Herkunft aus der handwerklichen Ausbildung und der Handwerkskultur (s. unten) ${ }^{29}$.

Doch es gab auch Differenzen zwischen den genannten Branchen. Denn die Erfahrungen, die im Bau- und Holzsektor gemacht wurden, unterschieden sich doch sehr von jenen Realitātsdeutungen in der metallverarbeitenden Industrie sowie im Maschinenbau.

Die Metaller (genauer: die gelemten Arbeiter aus der metallverarbeitenden Branche bzw. den Maschinenbauindustrien) dominierten in zahlreichen (regionalen) Parteizentren. In Düsseldorf machten die Metaller rund 35\% der Mitgliedschaft aus (1907) ${ }^{30}$. Ähnliches ist auch aus Augsburg, Berlin, Chemnitz, Stuttgart, Bielefeld und Nürnberg überliefert $^{31}$. Tatsāchlich hatten die Metaller in den metallverarbeitenden Branchen relativ gute Arbeitsplätze. Sie litten damals noch wenig unter einer Dequalifizierung der Arbeit; die Arbeitszeit betrug häufig „nur“ 10 Stunden. Oft genossen sie sogar eine relative Autonomie bei ihrer Arbeit, die meist in Werkstātten stattfand. Sie hatten noch

${ }^{27}$ Fischer, Intelligenz, 225.

${ }^{28}$ Allerdings sind die Statistiken schwer miteinander vergleichbar, weil in einem Teil von ihnen einzelne Berufe aufgezählt sind, während in einem anderen Teil die Statistik nach Branchen geordnet ist. Bei den Berufsanalysen muß zudem berücksichtigt werden, daß die Angaben nicht einheitlich vorgenommen wurden und daß sie vielfach über den tatsächlich ausgeübten Beruf des Mitglieds keine genaue Aussage zulassen. Die zeitlich umfassendsten und genauesten Angaben über die berufliche Zusammensetzung der Mitgliedschaft vermitteln wohl die von der Hamburger Sozialdemokratie vom Anfang der neunziger Jahre bis 1914 jährlich veröffentlichten Statistiken. Auszüge befinden sich in: Fricke, Arbeiterbewegung, 265.

${ }^{29}$ Nolan, Düsseldorf, 112

${ }^{30}$ Nolan, Düsseldorf, $104 \mathrm{ff}$. Allerdings sind hier keine genauen Zahlen angegeben.

${ }^{31}$ Für Bielefeld, Berlin, Chemnitz und Stuttgart siehe entsprechenden Hinweis bei Ditt, Bielefeld, 251; Fischer, Augsburg, 335. In Nürnberg betrug 1894 der Anteil 36\%, Rossmeisl, Nürnberg, 207. In München und Frankfurt, in Städten mit einer wirtschaftlichen Mischstruktur, lag der An- . teil der Parteimitglieder, die aus der Metallbranche stammten, bei 17 bzw. 20\%. Michels, Sozialdemokratie, 506; 500 (selbst errechnet). 
am ehesten ein starkes Selbstwertgefühl und progressive Zukunftsperspektiven. Ihre Berufsausbildung und ihre Arbeitskultur ermöglichten ihnen eine Identitätsbildung als qualifizierte Arbeiter und ein Bewußtsein ihrer relativ starken Position im industriekapitalistischen Produktionsproze $\beta^{32}$.

Lucas beschreibt noch einen anderen Typ eines Metallers. Auf Remscheid blickend charakterisiert er die Arbeiter in dem dort vorherrschenden kleineisen-produzierenden Gewerbe - vor allem sind die Feilenhauer hier zu nennen. Diese hätten es verstanden, sich durch zunehmende berufliche Spezialisierung der Proletarisierung zu widersetzen und die handwerkliche Tradition in einer wirtschaftlich kontinuierlich gewachsenen Mittelstadt aufrecht zu erhalten. Die Einbindung solcher Metaller in die SPD führt Lucas vorrangig auf die Kontinuität der Entwicklung und auf entsprechende Realitätsdeutungen zurück. Meist in traditional-patriarchalischen Sozialbeziehungen verwurzelt, ermöglichte ihre Erfahrungswelt ihnen eine auf Kontinuităten basierende rationale Zukunftsplanung, die eine relative Sicherheit bezüglich der eigenen Lebensperspektiven einschlo $\Omega^{33}$.

In vielen (wenn auch nicht in allen) Parteiprovinzen dagegen dominierten nicht die Metaller, sondern Handwerker aus den Branchen Stein/Bau und Holz, so z. B. in Göttingen (Stein/Bau: 27,4\%; Holz: 11,3\%) und in Marburg (Stein/Bau: 27\%; Holz: $23 \%)^{34}$. Zwar waren Handwerker aus diesen beiden Branchen auch in den Parteizentren stark vertreten, jedoch wurden sie dort nicht selten von den Metallern zahlenmāBig überrundet. Dies gilt auch für Hamburg ${ }^{35}$. Die stärkste Einzelgruppe innerhalb der Branchen Stein/Bau und Holz bildeten die Maurer, die beispielsweise in den drei sozialdemokratischen Vereinen in Hamburg 1907/08 2885 Personen ausmachten. (Das waren alleine schon $8,25 \%$ der gesamten Mitgliedschaft), gefolgt von den Tischlern mit 2664 Personen $(7,6 \%)^{36}$.

Die qualifizierten Arbeiter der beiden Sektoren Stein/Bau und Holz rekrutierten sich (wie die Metaller) überwiegend aus der Handwerkerschaft. Sie hatten - ebenfalls wie die Metaller - kaum mehr Hoffnung, jemals selbstāndige Meister ihres Faches zu werden. Auf Grund langer handwerklicher soziokultureller Traditionen machte es ihnen keine Probleme, in Organisationen eingebunden zu sein. Weder Mechanisierung noch Rationalisierung waren weit fortgeschritten. Im Unterschied zu der Situation bei den Metallern gab es aber in diesen Branchen noch viele Kleinbetriebe, die unter dem voranschreitenden Konzentrationsprozeß litten. Vor allem hatten die Kleinbetriebe

\footnotetext{
${ }^{32} \mathrm{Vgl}$. Nolan, Düsseldorf, 105 f.; für Augsburg: Fischer, Augsburg, 335.

${ }^{3}$ Über Remscheid siehe Erbard Lucas, Zwei Formen von Radikalismus in der deutschen Arbeiterbewegung (Frankfurt 1976) 250 ff.; künftig zitiert: Lucas, Zwei Formen.

${ }^{34}$ Die Zahlen sind entnommen aus: $t:$ Saldern, Einwohner, 116. In Singen spielten die Handwerker des Bau- und Ausbaugewerbes ebenfalls eine numerisch große Rolle in der Partei. Zang, Provinzialisierung, 22. Eine āhnliche Struktur der Parteimitgliedschaft ist auch aus Leipzig übermittelt. Dort waren die Branchen Stein/Bau und Holz 1904 mit 25,3 bzw. 12,1\% vertreten.

${ }^{35}$ Errechnet nach Fricke, Arbeiterbewegung, 258 if.

${ }^{36}$ Fricke, Arbeiterbewegung, $256 \mathrm{ff}$. Ähnlich verhielt es sich im schleswig-holsteinischen Wahlkreis, wo 1908 ebenfalls der Beruf des Maurers (mit 1239 Personen) in der Partei dominierte; ebda., $269 \mathrm{f}$.
} 
im Holzgewerbe stark gegen die maschinelle Konkurrenz größerer Betriebe anzukämpfen. Im Zuge der rapiden Urbanisierung und Industrialisierung zāhlte das Baugewerbe zwar zu den wachsenden Gewerbezweigen, allerdings erwies es sich auch als recht konjunkturempfindlich. Jede ökonomische Rezession wirkte sich im Baugewerbe überdurchschnittlich aus. Die Hochindustrialisierung brachte dieser Berufsgruppe also keine relative Stabilität ihrer Arbeitsplātze. Natürlich wußte man, daß Leute aus solchen Branchen immer irgendwo und irgendwann gebraucht wurden, aber vielfach fehlte wohl die Erfahrung der betrieblichen Konstanz: Die betrieblichen $\mathrm{Zu}$ kunftsperspektiven für solche Berufsangehörigen waren ungewisser als bei den Metallern. Hinzu kommt: Es mußte einen Unterschied ausmachen, ob man in den Leitsektoren industrieller Entwicklung (wie in den metallverarbeitenden Branchen) tätig war oder in den noch stark handwerksbestimmten, den Hochindustrialisierungsprozeß „begleitenden Branchen“ wie Stein/Bau und Holz. Allerdings konnten die relativ schwachen Unternehmer in den zuletzt genannten Branchen den Eintritt ,ihrer“ Arbeiter in die Gewerkschaften nicht verhindern, womit sich eine wichtige Chance zur Politisierung (s. unten) eröffnet ${ }^{37}$. Doch es gab in diesen Branchen auch Strukturen, die sich für eine Politisierung ungünstig auswirkten. Beispielsweise weiß man gerade von den Bauarbeitern, daß sie vielfach noch mit der ländlichen Lebenswelt verwachsen blieben (nicht zuletzt auf Grund häufig saisonaler Arbeitsverträge) und daß sie sich deshalb wohl auch nicht so leicht mit der urban-geprägten Arbeiterbewegungskultur identifizieren konnten wie die Metaller.

Wenn man also die partiellen Unterschiede zwischen den Branchen Metall einerseits und Stein/Bau sowie Holz andererseits herausarbeitet, und wenn man sich noch einmal in Erinnerung ruft, daß es gerade die Parteiprovinzen waren, in denen Stein/ Bau und Holz dominierten, während in Industrie- und Großstädten häufig die Handwerker aus der Metallbranche in der Partei den Ton angaben, so kann von daher gesehen auf sozio-strukturelle Unterschiede zwischen Parteiprovinzen und Parteizentren geschlossen werden: Zukunftsorientierte Selbstsicherheit (wie bei den Metallern in den Parteizentren) fehlte weitgehend in den Parteiprovinzen ${ }^{38}$. Dies lag freilich nicht so sehr am Beruf an sich - Maurer waren auch selbstbewußt und spielten in Großstädten eine wichtige Rolle (s. oben) - sondern mehr an der Kombination von Beruf und provinzieller Gesamtsituation.

Nun gab es auch Branchen, die auffallend schwach in der SPD vertreten waren. In diesem Zusammenhang müssen genannt werden: Bergbau, polygraphisches Gewerbe, Textil und Nahrung/Genuß ${ }^{39}$ sowie die metallerzeugenden Industriezweige.

\footnotetext{
${ }^{37} \mathrm{Vgl}$. dazu auch die UUberlegungen bei Nolan, Düsseldorf, 110.

${ }^{38} \mathrm{Zu}$ diesem Ergebnis kommt man auch, wenn man die internen Parteiprotokolle der Göttinger SPD auswertet. Es gibt allerdings immer auch Ausnahmen, z. B. bestimmte ländliche Gebiete in Sachsen (s. G. A. Ritter in diesem Band) oder die Dominanz der Metaller beispielsweise in der Provinzstadt Rheine. (Diesen Hinweis verdanke ich Friedhelm Boll.)

${ }^{39}$ Bācker und Fleischer konnten z. B. in Göttingen für die Partei kaum gewonnen werden. Zum Teil wohnten sie noch im Haus des Arbeitgebers, $z$. T. hofften sie auf einen spāteren beruflichen Aufstieg oder gar auf Selbständigkeit. Allein die Zigarrenarbeiter galten als organisationsfreudig und machten in der Göttinger SPD 8,6\% der Parteimitgliedschaft aus.
} 
Betrachten wir uns einige dieser Sektoren etwas genauer:

Interessanterweise schlossen sich die Drucker selten der SPD an. Obwohl in Düsseldorf 1200 Drucker in der Gewerkschaft organisiert waren (1907), erklärten nur 51 ihren Beitritt zur SPD $^{40}$. In Hamburg machten die Drucker nur 1,2\% der Mitglieder aus (1907/08: 416 Personen) ${ }^{4}$. Als Arbeiterelite und überzeugt von der Unersetzbarkeit ihrer Fähigkeiten glaubten sie wohl, daß ökonomische Kämpfe alleine ausreichen würden. Allerdings gibt es offensichtlich auch hier Unterschiede zwischen Parteizentren und Parteiprovinzen. Es ist auffallend, daß diese Branche in Marburg (1906) 19\% der Mitglieder stellte ${ }^{42}$. In Göttingen machten die Beschäftigten aus der polygraphischen Branche zwar nur 7,5\% der Mitglieder aus (1908: 14 Personen), doch ist bemerkenswert, daß immerhin (1907) 6,1\% aller in dieser Branche Beschäftigten in der Partei organisiert waren, das war - verglichen mit den anderen Branchen am Ort - der höchste Prozentsatz ${ }^{43}$.

Bei der metallerzeugenden Branche wirkten sich offenbar betriebliche Großstrukturen sowie die spezifische Arbeitssituation für einen Eintritt in die SPD ungünstig aus, insofern die Aufnahme informeller Kontakte während der Arbeit erschwert war. Damit entfiel eine wichtige Voraussetzung für die Politisierung. Dort waren die Arbeitszeiten auch länger (12-Stunden-Schicht im Unterschied zum 10 Stundentag im Maschinenbau sowie in den metallverarbeitenden Industrien); außerdem unterlag die Arbeit auf Grund des starken technologischen Wandels schon damals einer Intensivierung ${ }^{44}$. Hinzu kam die höchst autoritäre Betriebsführung und der sogenannte Sozialpaternalismus (firmeneigenes Sozial- und Kulturprogramm), wodurch die Voraussetzungen für politische Organisationen ebenfalls verschlechtert wurden. Die häufigste Protestform dieser Arbeiter war nicht der Eintritt in die Gewerkschaften und in die SPD, sondern das Verlassen der Firma. Dies gilt vor allem für die ungelernten Arbeiter in der Eisen- und Stahlfabrikation.

Ein schlechtes Rekrutierungsfeld gab auch die Branche Textil/Bekleidung ab. In Göttingen machte diese Berufsgruppe (Leder- und Textilbranche) in der SPD zwar $11,8 \%$ aus, das waren jedoch nur 2,3\% der in dieser Branche (1907) Beschäftigten. Bei den Mitgliedern handelte es sich fast ausschließlich um die von jeher stark überbesetzten Berufe der Schneider und Schuhmacher, nicht aber um die Gruppe der Textilarbeiterinnen ${ }^{45}$. Die vor allem in der Textilindustrie beschäftigten Frauen ließen sich schwerer als ihre männlichen Kollegen organisieren (s. unten). In Augsburg gelang es überdies den Textilunternehmern, scharfe Kontrollen bei Neueinstellungen durchzuführen. In der Textilindustrie habe es „Furcht vor Repressalien“" gegeben, und die viel-

40 Nolan, Düsseldorf, 111.

${ }^{1}$ Fricke, Arbeiterbewegung, 257.

42 Michels, Sozialdemokratie, zitiert aus: 1 : Saldern, Einwohner, 116.

43 : Saldern, Einwohner, 116.

${ }_{44}^{4}$ Dazu Nolan, Düsseldorf, 106f. In Düsseldorf waren annāhernd 50\% der Eisen- und Stahlarbeiter in Betrieben mit mehr als 500 Beschäftigten angestellt, während es bei den Maschinenbauern nur $25 \%$ waren.

4s 2 Saldern, Einwohner, 119. 
seitigen „Abhāngigkeiten durch das betriebliche Wohlfahrtsystem“ ließen „ein politisches Engagement nicht ratsam erscheinen ${ }^{\text {"46. }}$.

\section{Sozio-kulturelle Dispositionen}

Die bisherigen Ausführungen über die soziale und berufliche Zusammensetzung der sozialdemokratischen Mitgliedschaft stellten nur eine erste grobe, an äußeren Merkmalen orientierte Analysc dar. Sozio-kulturelle Erklärungsmuster für den Parteieintritt (1), aber auch für die Ablehnung der SPD bzw. für die Gleichgültigkeit gegenüber der Partei (2) müssen hinzukommen. Schließlich soll auch noch kurz auf die besondere Situation der Frauen eingegangen werden (3).

\section{Positiv wirkende Einflußfaktoren ${ }^{47}$}

Im folgenden werden einige sozio-kulturelle Voraussetzungen thematisiert, die einen Parteieintritt begünstigten.

\section{a) Bildung und Ausbildung}

Aus Autobiographien von Sozialdemokraten kann man Faktoren erkennen, die offensichtlich häufig einen Politisierungsprozeß bewirkten, der schließlich zum Eintritt in die SPD führte ${ }^{48}$. Neben der ,richtigen“ Lektüre, der Überzeugungsarbeit durch Eltern (vor allem ist an die Parteimitglieder der zweiten Generation zu erinnern), durch Freunde, Bekannte, Kollegen ${ }^{49}$ sowie der Ausstrahlungskraft sozialdemokratischer Führer, spielte die handwerkliche Ausbildung eine große Rolle. Dadurch erhielt der Arbeiter offenbar ein gewisses Selbstwertgefühl, wodurch seine Fähigkeiten vergrößert

${ }^{46}$ Fischer, Augsburg, 336.

${ }^{47}$ Zum Folgenden siehe auch die 54 Kurzbiographien bei $v$. Saldern, Arbeiter-Reformismus, $290 \mathrm{ff}$. Im folgenden ein Beispiel aus Nolan, Düsseldorf, $108 \mathrm{f}$.: Es handelt sich um die Sozialbiographie des Kunsttischlers und regionalen Parteiführers Berten: Berten wuchs in einem kleinen rheinischen Dorf auf, wo sein Vater eine kümmerliche Existenz als Zimmerer fristete. Schon als Kind half er seinem Vater, und nach der Volksschule lernte er das gleiche Handwerk. Im Jahre 1891, als Berten 18 Jahre alt war, begab er sich auf die Reise, zumal die Berufsaussichten auf dem Lande āußerst gering waren. Am Niederrhein zog er von Stadt zu Stadt, erweiterte seinen Wissensstand und lernte das städtische proletarische Leben kennen. Als er in Dortmund eine Beschäftigung fand, trat er der Gewerkschaft bei. Einige Jahre später, nachdem er immer wieder an politischen Diskussionen auf Gewerkschaftstreffen teilgenommen hatte, schloß er sich schließlich auch der SPD an.

${ }^{48} \mathrm{Vgl}$. Jochen Loreck, Wie man früher Sozialdemokrat wurde. Das Kommunikationsverhalten in der deutschen Arbeiterbewegung und die Konzeption der sozialistischen Parteipublizistik durch August Bebel (Schriftenreihe des Forschungsinstituts der Friedrich-Ebert-Stiftung 130, BonnBad Godesberg 1977) 159 ff.; Loreck berichtet überdies von Leuten, die in die Partei eintraten, weil ihnen andere Aufstiegsmöglichkeiten versperrt waren. So wollte Bernstein studieren, Blos wollte Philologe werden; Bromme und Löwe träumten vorm Lehrerberuf, Keil und Noske wären gerne Förster geworden, und Severing liebāugelte mit dem Beruf eines Pfarrers. Weitere Beispiele: ebda., 137. Insgesamt enttāuscht die Arbeit aber wegen der geringen Interpretationskraft.

${ }^{49}$ Eine gute Voraussetzung stellte auch die Mitgliedschaft in der Arbeiterjugendorganisation dar. 
wurden, über seinen individuellen Lebenshorizont hinauszutreten und zu erkennen, $\mathrm{da} ß$ sich die Interessen der Arbeiterklasse nur in autonomen Arbeiterorganisationen vertreten ließen. Auf dieser Basis konnten dann auch unter Umstānden die im 1. Kapitel thematisierten Faktoren (Schicht/Beruf) wirksam werden und sich gewisse Utopiebedürfnisse nach einem zukünftigen „besseren Leben“ in einer besseren (sozialistischen) Gesellschaft entfalten ${ }^{50}$. Im Unterschied dazu fiel es Arbeitern ohne (Aus-)Bildung (die im übrigen auch häufig große materielle Sorgen hatten) oftmals schwer, über die reinen gegenwartsbezogenen Reproduktionsinteressen der eigenen Existenz und der ihrer Familien hinauszukommen ${ }^{51}$.

\section{b) Alter}

In der Literatur wird übereinstimmend ausgesagt, daß die meisten Parteimitglieder ein Alter zwischen 20 und 40 Jahren aufwiesen ${ }^{52}$. Die SPD war also in wilhelminischer Zeit keineswegs überaltert - im Unterschied zur Phase der Weimarer Republik. Die meisten Sozialdemokraten standen beim Eintritt in die Partei auf dem Höhepunkt ihres Lebenszyklus. Sie hatten bereits eine gewisse Berufssicherheit erreicht, und ihr Verdienst lag (verglichen mit älteren und ganz jungen Kollegen) relativ hoch. Viele waren überdies verheiratet und hatten Kinder, für die sie eine bessere Zukunft erkämpfen wollten.

\section{c) Gewerkschaften}

Die Not, die Arbeiter bei sich und bei anderen erfuhren, deuteten viele als Auswirkung der Klassengesellschaft ${ }^{33}$. Gleichzeitig erlebten Arbeiter, wie man durch gemeinsamen Einsatz Verhältnisse zumindest partiell verbessern konnte. Erfahrungen sammelten sie hierbei meist im Rahmen gewerkschaftlicher Tätigkeiten und Streiks. Viele traten deshalb zunächst in die Gewerkschaft und später erst in die Partei ein. In der Literatur wird aus diesem Grunde die Bedeutung der Gewerkschaften als Politisierungsmedium übereinstimmend herausgestellt ${ }^{54}$. Ja, man kann vereinfacht sagen: je stärker die Gewerkschaften am Orte waren, desto stärker war die SPD 55 . Dies gilt z. B. auch für Remscheid. E. Lucas weist in seinem Buch über den Arbeiterradikalismus auf die relativ großen gewerkschaftlichen Erfolge der Remscheider Arbeiter hin. So konnten Tarifverträge durchgesetzt und die betriebliche Zersplitterung in viele Klein- und Kleinstbetriebe aufgefangen werden. Dieser Erfolg gewerkschaftlicher Politik habe

30 Walter Caroli/Robert Stimpel, Geschichte der Lahrer SPD. Ein Beitrag zur politischen Entwicklung in der Stadt Lahr (Lahr 1979) 91.

"1) Vgl. Fricke, Arbeiterbewegung, 272.

32 Ebda.; Rossmeisl, Nümberg, 210; Nolan, Düsseldorf, 118, $120 \mathrm{f}$; Wuerth, Göppingen, $59 \mathrm{f}$.

${ }^{33}$ Bezüglich der Deutung der Gesellschaft als Klassengesellschaft vgl. $v$ : Saldern, Arbeiter-Reformismus, $58 \mathrm{ff}$.

${ }^{4}$ Z. B. Ditt, Bielefeld, 251; Jörg Schadt, Wolfgang Schmierer, Die SPD in Baden-Württemberg und ihre Geschichte. Von den Anfängen der Arbeiterbewegung bis heute (Schritten zur politischen Landeskunde Baden-Württembergs 3, Stuttgart 1979) 82; im folgenden zitiert: Schadt, Scbmierer, Baden-Württemberg. Wuerth, Göppingen, $59 \mathrm{f}$. Oft wirkten auch sog. Vorfeldvereine als Politisierungsmedium.

${ }^{55}$ Vgl. z. B. Guttsman, Social Democratic Party, 155. Dort nähere Angaben. 
sich offenbar auch für die SPD ausgezahlt: Remscheid wurde eine sozialdemokratische Hochburg mit einem gut ausgebauten sozialdemokratischen Organisationswesen (Zeitung, Konsumverein, Volksbibliothek) ${ }^{56}$.

Doch würde das komplizierte Beziehungsgeflecht von Gewerkschaften und SPD vereinfacht werden, würde man die Gewerkschaften nur unter dem Aspekt eines sozialdemokratischen Rekrutierungsfeldes sehen wollen. Schließlich darf nicht vergessen werden, daß noch um 1900 die Zahl der Parteimitglieder höchstens ein Drittel der in den Freien Gewerkschaften organisierten Arbeiter ausmachte ${ }^{57}$. Es ist in diesem Zusammenhang auch daran zu erinnern, daß zwischen Gewerkschaften und Partei Spannungen bestanden ${ }^{58}$, die erst mit der zunehmenden „Vergewerkschaftung“ der Partei abnahmen. Schließlich waren sozialdemokratische Mitglieder um 1905 „fast durchweg auch Gewerkschaftler"59, während viele Gewerkschafter nach wie vor nicht den Sprung in die Partei fanden.

Beachtenswert ist auch die Tatsache, daß der Anteil der Gewerkschaftsmitglieder in der sozialdemokratischen Mitgliedschaft von Ortsverein zu Ortsverein beträchtlich schwankte. Die Spannweite lag zwischen rund 10\% in Frankfurt (um 1905) und ca. $40 \%$ in Bielefeld (1909) sowie in Hamburg $(1911 / 12)^{60}$. Schönhoven führt die regionalen und lokalen Unterschiede nicht nur auf strukturelle Merkmale, sondern auch auf Art und Intensität der Mitgliederwerbung zurück ${ }^{61}$. Wichtig ist ferner in diesem Zusammenhang die jeweilige „Kultur der Arbeit“, wozu auch Qualitātsbewußtsein (über die Produkte und die Tätigkeit als ursprüngliche Produzenten) sowie Solidarităt gehörten. Ihre unterschiedliche Ausprägung hatte sicherlich auch Einfluß auf die Entwicklung der Anzahl der gewerkschaftlich organisierten Partei-Mitglieder (s. auch oben).

\section{d) Zuwanderer}

Nolan betont in ihrer Studie über die Sozialdemokratische Partei in Düsseldorf die große Bedeutung, die die Zuwanderer für die SPD gehabt hätten ${ }^{62}$. (Vor allem gelte ${ }^{56}$ Lucas, Zwei Formen, $92 \mathrm{ff}$.

37 Michels, Sozialdemokratie, 490. Von den 360000 Mitgliedern der Metallarbeitergewerkschaft war auch nur ein Viertel in der Partei. Guttsman, Social Democratic Party, 156. Erinnert sei in diesem Zusammenhang noch daran, daß es 1893 in der Partei zu einem sehr umstrittenen Beschluß gekommen ist, der darauf hinauslief, daß jeder Parteigenosse in die Gewerkschaften eintreten müsse. Micbels, Sozialdemokratie, $491 \mathrm{f}$.

${ }^{58}$ Ebda.

59 Ebda.

${ }^{60}$ Die gewerkschaftlich organisierten Frankfurter Buchdrucker waren besonders wenig in der Partei zu finden. Der Anteil betrug 6\%. Michels, Sozialdemokratie, 499; Ditt, Bielefeld, 251; Volker Ullrich, Die Hamburger Arbeiterbewegung am Vorabend des ersten Weltkrieges bis zur Revolution 1918/19 (Diss. phil., Hamburg 1976) 78; weitere Zahlen: Göttingen 14\%, Marburg 23\%; vgl. v. Saldern, Einwohner, 173.

${ }^{61}$ Klaus Scbönboven, Expansion und Konzentration. Studien zur Entwicklung der Freien Gewerkschaften im wilhelminischen Deutschland 1890-1914 (Stuttgart 1980) 107 ff., 118.

${ }^{62}$ Vgl. z. B. Nolan, Düsseldorf, 99. In der ersten Generation der lokalen Sozialdemokratie spielte die Ansässigkeit der Genossen eine große Rolle. So hatten die „fliegenden Agenten“ der Lassalleschen Organisation hāufig dann keinen dauerhaften Erfolg, wenn sie nicht ausreichend von Einheimischen unterstützt wurden. 
dies für die Metaller; s. auch oben.) Die meisten von ihnen stammten aus dem Rheinland, viele kamen aus der Region Düsseldorf ${ }^{63}$. Solche Arbeitergruppen seien bereit gewesen, das städtische Leben und die urbane Kultur auf sich wirken zu lassen ${ }^{64}$. Sie hātten sich gegenüber den politischen Ideen besonders aufgeschlossen gezeigt und mit ihrer Vergangenheit weitgehend gebrochen. In einer katholischen Stadt wie Düsseldorf seien vor allem die protestantischen Zuwanderer als Rekrutierungsfeld für die SPD wichtig gewesen, weil diese von der lokalen dominierenden katholischen Kultur von vornherein ausgeschlossen waren. Die (protestantischen) Zuwanderer hätten sich an industrieller Arbeit interessiert gezeigt, nach relativ gut bezahlten Arbeitsplätzen gesucht, wo sie ihre fachlichen Kenntnisse unter Beweis stellen konnten und ihnen eine relative Autonomie geboten wurde. Ihre zum Teil radikale Kapitalismuskritik basierte also auf einer spezifischen Form vorlaufiger „Anerkennung“ der kapitalistischindustriellen Entwicklung - nämlich als Durchgangsstadium zu einer besseren (sozialistischen) Gesellschaft ${ }^{65}$.

\section{e) Parteizentren - Parteiprovinzen}

Wer in (regionalen) Parteizentren tătig war, dem fiel die Entscheidung, in die Partei einzutreten, leichter als jenen, die in Parteiprovinzen lebten. Dies gilt vor allem für Arbeiter, die nicht nur in Parteizentren, sondern darüberhinaus auch noch in bestimmten Stadtquartieren (SPD-Hochburgen) wohnten ${ }^{66}$ oder in Betrieben arbeiteten, in denen die Gewerkschaften gut vertreten waren. In den Parteizentren fand man ein sehr viel größeres Angebot an sozialdemokratischen Selbsthilfeorganisationen, Wirtshäusern (evtl. sogar Volksheimen) sowie Zeitungen vor. Streik- und Demonstrationsöffentlichkeiten führten zumindest zu einer partiellen Beherrschung des öffentlichen Raumes. Die sozialdemokratische Kultur innerhalb und außerhalb der Parteiorganisation konnte insgesamt gesehen eine ungleich stärkere Auswirkung und damit Anziehungskraft auf Unentschlossene ausüben als dies in den Parteiprovinzen möglich war,

\footnotetext{
${ }^{63}$ Das waren $52 \%$ von insgesamt 141000 Zuwanderem im Jahre 1905 . Westfalen stellte $15 \%$ der Ankömmlinge. 28\% zogen von weiter entfernt liegenden Gegenden zu, einige sogar aus dem Ausland. Ein Teil der Zuwanderer lebte ehedem in Gebieten, die ökonomisch weniger gut entwickelt waren als Düsseldorf, ein anderer Teil stammte aus Städten mit einer vergleichbar entwickelten industriellen Struktur, aber mit niedrigerer Lohnskala. Eine knappe Mehrheit von $\mathrm{Zu}$ wanderem kam aus kleinen und mittelgroßen Städten, während nur ein Drittel in lāndlichen Gebieten geboren war. Ein überraschend hoher Prozentsatz (15) stammte von Stādten mit mehr als 100000 Einwohnern. Schließlich gab es noch welche ( $11 \%$ der Zuwanderer), die vorher in einer größeren Stadt, als Düsseldorf es war, lebten. Nolan, Düsseldorf, 114.

${ }_{64}$ Hierzu und zum Folgenden ebda., 116, vgl, auch 114.

${ }^{65} \mathrm{Vgl}$. auch ebda., 117.

${ }^{66}$ Eine (große) Bedeutung haben wohl auch die Wohnquartiere für die Entscheidung zugunsten des Eintritts in die SPD gehabt. Bekanntlich gab es in den Städten Wohnviertel, die als (Wahl-) Hochburgen der SPD galten. Ein Problem für die Forschung liegt freilich darin, herauszufinden, inwieweit spezifische Kommunikationsformen im Wohnquartier hierbei eine Rolle gespielt haben. Hinzuweisen ist auch noch auf die Tatsache, daß viele Arbeiterfamilien zwar mobil waren, aber die Mobilităt sich in rāumlich engen Grenzen hielt: Man zog meist nur innerhalb des Viertels um. Hierzu einschlägig und differenzierend: Stepban Bleek, Mobilität und Seßhaftigkeit in deutschen Großstādten wāhrend der Urbanisierung, in: Geschichte und Gesellschaft 15 (1989) $5-34$.
} 
wo es an verdichteter Urbanität und sozialdemokratischem Milieu fehlte. In den Parteizentren konnte ein Stück weit die kulturelle Hegemonie des stādtischen Bürgertums durchbrochen und proletarische Öffentlichkeit hergestellt werden. In diesem Zusammenhang vermochte auch ein neu aufgebauter Parteiapparat eine vorteilhafte Rolle zu spielen (vgl. Boll, S. 102) ${ }^{67}$, kurzum, die Erfahrungsmöglichkeiten und der Erfahrungshorizont der in Parteizentren lebenden Arbeiter war gefächerter und vielseitiger als in den Parteiprovinzen.

Dort in den Parteiprovinzen war die sozialdemokratische Organisation oftmals schwach; gleichzeitig wirkte die kulturelle bürgerliche Hegemonie mit ihren scheinbaren Integrationsangeboten auch an die Arbeiter vielfach noch erdrückender als in den Parteizentren. Ein von der Partei getragenes sozio-kulturelles Gegenlager konnte allenfalls nur punktuell entwickelt werden. Ein intellektuelles Milieu, das für die Entwicklung der SPD hätte unter Umstānden förderlich sein können, fehlte dort meistens. Auch wer mit den traditionellen Lebensformen und Realitătsdeutungen gebrochen hatte, blieb mit der agrarisch-vorindustriellen Welt realiter stārker verbunden, als dies in den Parteizentren der Fall war. Gleichzeitig erlebte die Parteiprovinz jedoch auch entscheidende Veränderungen ihres Erfahrungshorizontes durch die Industrialisierung. Beide Lebenswelten - die agrarisch-handwerkliche und die industriell-handwerkliche - überlagerten sich, standen in mehrdimensionaler spannungsgeladener Verflechtung zueinander ${ }^{68}$. Wichtig - aber noch recht unerforscht - ist die Frage, inwieweit ein Zusammenhang zwischen der mehr populistischen Ausprägung eines sozialdemokratischen Ortsvereins (z. B. im Westen Münchens) und der erfolgreichen quantitativen Entwicklung der Partei bestanden hat ${ }^{6 \%}$.

\section{Sozio-kulturelle Hemmnisse}

Die Erklärungsmuster, die für die Analyse der Parteieintritte förderlich waren, erhalten noch schärfere Konturen, wenn auch die gegenläufigen Dispositionen, die einen Eintritt in die Partei erschwerten, vorgestellt werden ${ }^{70}$, soweit sie nicht schon im vorherigen Abschnitt als Kontrastmuster Erwähnung fanden.

67 Eine dominierende Stellung nahm z. B. der 8. Hannoversche Reichstagswahlkreis, der die Städte Hannover und Linden sowie den Landkreis Hannover umfaßte, in der Provinzialorganisation ein. Von den 12 zu dieser Organisation gehörenden Wahlkreisen rekrutierte der Wahlkreis Hannover-Linden im Jahre 1907 65,7\% und 1913 57,6\% der gesamten Mitglieder. Boll, Massenbewegungen, 103. In diesem Zusammenhang ist auch die Frage wichtig, inwieweit es zu einer Tiefenverankerung der Partei am jeweiligen Ort kam - beispielsweise durch entsprechende Milieus.

${ }^{68}$ Zang, Provinzialisierung, passim. Vgl. auch Adelbeid v. Saldern, Arbeiterradikalismus - Arbeiterreformismus. Zum politischen Profil der sozialdemokratischen Parteibasis im Deutschen Kaiserreich. Methodisch-inhaltliche Bemerkungen zu Vergleichsstudien, in: Internationale wissenschaftliche Korrespondenz zur Geschichte der Deutschen Arbeiterbewegung (IWK), 20. Jg. H. 4 (1984) 483-498.

${ }^{69}$ Diesen Hinweis verdanke ich Stephan Bleek. Unter solcher Fragestellung ist auch das Verhāltnis von Arbeiterbewegungskultur und Popularkultur wichtig. (Für die 1920er Jahre kommt noch das Verhältnis zur neuen Massenkultur hinzu.)

${ }^{70}$ Auf die nationale Frage als Einflußfaktor wird hier nicht weiter eingegangen. 
a) Begrenzter Stadtaufenthalt

Nolan weist in diesem Zusammenhang vor allem auf die nur zeitweise in die Stadt ziehenden (häufig un- bzw. angelernten) Arbeitergruppen hin. Sie hätten Organisationen wie SPD und Gewerkschaften mit Indifferenz oder gar Feindseligkeit betrachtet. Gegenüber ihrer eher trostlosen (oftmals ungelernten) Berufstätigkeit hätten sie eine instrumentelle Haltung an den Tag gelegt. Im Grunde wollten sie sowohl der industriellen Arbeit als auch dem städtischen Leben entkommen, und nur wenn dieses Entkommen nicht mehr möglich war, habe es eine Chance gegeben, sie für die SPD zu mobilisieren ${ }^{71}$.

b) Sozialkatholisches Milieu

Ein zweiter Typ des für die SPD nur schwer erreichbaren Arbeiters war jener, der im sozialkatholischen Milieu eingebunden blieb, im Falle Düsseldorf also die einheimischen Arbeiter. Die einheimischen Arbeiter seien - so wieder Nolan - in einer streng katholischen Umgebung aufgewachsen, also ganz im katholischen Milieu integriert gewesen. Wenn diese Leute sich der Sozialdemokratie anschließen wollten, mußten sie nicht nur mit der Kirche, sondern auch mit ihrer eigenen Vergangenheit und Gegenwart brechen, d.h. mit ihrer bisherigen Lebensweise und ihrer katholischsozialen Vision ${ }^{72}$. Dies taten eben nur wenige. Zwischen 1896 und 1902 seien nur 53 Einheimische der Düsseldorfer SPD beigetreten. Erst als in den späten wilhelminischen Jahren der Konservatismus in der Zentrumspartei stärker hervortrat, seien weitere Arbeiter diesem Sozialmilieu entfremdet worden ${ }^{73}$. Vom protestantischen Lager her war offensichtlich der Entschluß, in die SPD einzutreten, leichter, wobei der Loslösungsprozeß (Entkirchlichung) häufig schrittweise erfolgte ${ }^{74}$.

\section{c) Überdimensionaler Kultursprung}

Ein anderer Typ eines „Nicht-Sozialdemokraten“ kennzeichnet Lucas am Beispiel des Hamborner Arbeiters. Dieser war in konzentrierten Großunternehmen der Montanindustrie in einer aus dem Boden gestampften Arbeitergroßstadt beschäftigt. Die dort arbeitenden Menschen hatten meist eine agrarische Herkunft, waren nach Hamborn zugewandert, wiesen also eine starke Diskontinuitāt in ihrer individuellen Lebensgeschichte auf, gepaart mit einer unsicheren Zukunft. Ihre Arbeitswelt war vom Großkapital bestimmt und von entfremdeter Anonymität des Arbeitsplatzes geprägt, ihre Zielperspektiven richteten sich nur auf das Unmittelbare und auf das Heute ${ }^{75}$. Ein Vergleich mit den nach Düsseldorf zugewanderten handwerklich ausgebildeten Arbeitern, die in der metallverarbeitenden Branche landeten (s. oben), bietet sich an:

${ }^{71}$ Nolan, Düsseldorf, 115, vgl. auch 114, $105 \mathrm{f}$.

${ }^{72} \mathrm{Vgl}$. Robe in diesem Band.

${ }^{73}$ Nolan, Düsseldorf, 113. So ist auch die antikirchliche Haltung der SPD zu verstehen.

${ }^{74}$ So waren die Göttinger Sozialdemokraten vielfach noch Mitglied der evangelischen Kirche geblieben; i: Saldern, Arbeiter-Reformismus, $290 \mathrm{f}$. Über das Verhältnis Religion - Sozialdemokratische Bewegung arbeitet derzeit Wilfried Spobn, Berlin. Ihn interessiert vor allem die Frage der Transformation religiösen Gedankenguts in die Arbeiterbewegung.

${ }^{75}$ Lucas, Zwei Formen, $280 \mathrm{ff}$. 
Der Unterschied lag offensichtlich darin, daß der nach Düsseldorf zugewanderte (spātere) Sozialdemokrat über eine handwerkliche Tradition verfügte und eine individuelle Ausbildung hinter sich gebracht hatte und schließlich einen relativ guten Arbeitsplatz fand. Den Zuzug in die Stadt mögen diese Arbeiter vielleicht auch als eine Art Befreiung empfunden haben. Demgegenüber kannte der nach Hamborn Zugewanderte meist nicht den handwerklichen Lebens- und Arbeitszusammenhang aus eigener Erfahrung; die Industrialisierung erlebte er nicht prozeßhaft, sondern sprunghaft und zugleich in ihrer ausgeprägtesten und darüberhinaus höchst anonymen Form. Der abrupte Übergang von der agrarisch-orientierten Alltagswelt zur großindustriellen Arbeitsstruktur sei meist nur als passiv zu erleidendes Schicksal erfahren worden, das allerdings bei einer bestimmten Neukonstellation durchaus in (parteiungebundene) politische Radikalität umschlagen konnte, wie dies tatsächlich bei den Hamborner Arbeitern 1918/19 der Fall war ${ }^{76}$.

d) Die Pendler (und Arbeiterbauern)

Eine andere Gruppe von Arbeitern, die meist nichts von der Partei wissen wollte, stellten die Pendler dar. In den Worten von Wuerth: „Viele dieser Arbeiter waren oft eifrig darauf bedacht, ihr Geld in Grundbesitz anzulegen oder arbeiteten auf den Tag hin, an dem sie als selbständige Handwerker die Fabrik verlassen konnten. Diese ländlichen Gemeinden boten den Sozialdemokraten keine Flucht in die Anonymität; ihre gesellschaftliche Struktur richtete sich gegen die Beteiligung an der Arbeiterbewegung und da es örtliche Gewerkschafts- und Parteiorganisationen in den meisten Dörfern nicht gab, war es für die Arbeiter schwierig, durch kollektive Bemühungen ihren Arbeitsplatz zu sichern. ${ }^{477}$

Hãufig handelte es sich bei den Pendlern eben auch um Arbeiterbauern, vor allem in Württemberg. Viele wohnten noch auf dem Lande und gingen nur zur Arbeit in die Stadt, blieben so mehr oder weniger in die traditionell-dörfliche Lebensweise eingebunden. Gerade an Württemberg kann man überdies das Stadt-Landgefälle aufzeigen, insofern sich der Mitgliederzuwachs der SPD bis 1914 auf die Städte der „Industriestraße" (von Heilbronn bis Göppingen) konzentrierte, wo 1913 etwa 4/5 der württembergischen sozialdemokratischen Mitglieder lebten ${ }^{78}$.

e) Sozialdemokratischer Wähler in der Parteiprovinz

Ein weiterer Typ eines für die SPD schwer ansprechbaren Arbeiters war der sozialdemokratische Wāhler in einer Parteiprovinz wie Göttingen, in der die bürgerliche kulturelle Hegemonie in besonderer Weise dominant war. In Göttingen wurde die bürgerliche Hegemonie nicht nur durch städtisches Bürgertum, sondern auch durch Universität und Garnison repräsentiert. Dort - wie sicherlich auch anderswo in Partei-

${ }^{76}$ Die Hamborner Arbeiter neigten 1918/19 zu mehr spontanen, aber keineswegs unorganisierten Aktionsformen. Lucas führt diese Tatsache nicht zuletzt auf den agrarischen Mentalitātshorizont dieser Arbeitergruppe zurück. Lucas, Zwei Formen, $250 \mathrm{f}$.

77 Wuerth, Göppingen, 57.

78 Über die Arbeiterbauern siehe Schadt/Schmierer, 10. 
provinzen - hatte man noch nach 1890 vielfältige soziale Diskriminierungen zu befürchten, wenn man sich offen als SPD-Mitglied zu erkennen gab. So waren im Jahre 1907 im 12. Reichstagswahlkreis (in und um Göttingen) nur rund 4\% der Wähler in der SPD organisiert; dieser Anteil stieg 1913 zwar auf 13,2\%, aber - verglichen mit den Parteizentren - war dies wenig: In den Groß- und Industriestädten (vor allem in protestantischen und industriellen Distrikten) machte der Anteil der sozialdemokratischen Mitglieder an der SPD-Wāhlerschaft bis zu 50\% aus ${ }^{79}$. Durch die Analyse der städtisch und ländlich gemischten Gebiete (so im 6. Wahlbezirk Schleswig-Holstein) kann dieser Unterschied besonders deutlich herausgearbeitet werden: Im Durchschnitt betrug der Anteil der sozialdemokratischen Mitglieder an der sozialdemokratischen Wählerschaft (1907) 42,5\%, die ortsbezogene Spannweite lag jedoch zwischen 18 und $65 \%$, war also immens gro $\beta^{80}$. In der Parteiprovinz fand man folglich besonders häufig jenen Arbeitertyp vor, der trotz seiner Sympathie für die SPD nicht aus seiner Anonymität heraustreten wollte. Erst nach 1918/19 ānderte sich die Situation, weil ein offenes Bekenntnis zur SPD keine Repressalien mehr nach sich zog ${ }^{81}$.

\section{f) „Kultur der Armut“}

Mit diesem von Oscar Lewis geprāgten Ausdruck soll das Leben jener unteren (Arbeiter-)Schichten ins Blickfeld rücken, die nicht ständig in den Produktionsprozeß eingebunden waren. Für unseren Zusammenhang ist wichtig, daß diese Bevölkerungsschichten (Gelegenheitsarbeiter, Saisonarbeiter etc.) zwar u.U. ein starkes Protestpotential entwickelten - bekannt sind tumultöse und gewaltsame Aktionsformen, - daß dieses Protestpotential jedoch meist unorganisiert blieb und sich weitgehend außerhalb der sozialdemokratischen Arbeiterbewegung artikulierte. Grüttner hebt mit Recht hervor, daß mobile, ungelernte Arbeiter nur schwer dauerhaft organisierbar waren. „Die von der Sozialdemokratie angebotene Perspektive einer langsamen und kontinuierlichen Verbesserung der proletarischen Lebenslage durch den geduldigen Aufbau machtvoller Organisationen blieb diesen Arbeitern fremd, da sie quer lag zu ihrem eigenen, durch Diskontinuität geprägten, augenblicksorientierten Lebensstil. Außerdem bezogen die Handwerksgesellen und Facharbeiter, die die Arbeiterorganisationen hauptsāchlich trugen, einen Teil ihres Selbstwertgefühls gerade auch aus der demonstrativen Distanz gegenüber den unteren Arbeiterschichten, dem „Lumpenproletariat“. Die diskriminierende Behandlung der Armen durch Polizei und andere staatliche Institutionen hat dazu beigetragen, diese Kluft weiter zu öffnen.“" ${ }^{\text {(22 }}$

79 In Bremen war der Anteil besondes hoch (1913: 40\%); Guttsman, Social Democratic Party, 153. Der Prozentsatz der Parteimitglieder an der Wählerschaft betrug im Deutschen Reich 1906: 13, 1909: 19,1 und 1913: 23,1. Protokoll über die Verhandlungen des Parteitags der SPD 1910.

${ }^{80}$ Guttsman, Social Democratic Party, 153; große Unterschiede stellt auch G. A. Ritter für Sachsen fest. Dort kam es seit 1903 zu einem Schub in der Mitgliederentwicklung (Nachholeffekt); vgl. G. A. Ritter in diesem Band. In diesem Zusammenhang spielt vielleicht auch das „Alter" des Wahlkreises eine Rolle.

${ }^{81}$ Vgl. dazu Boll, Massenbewegungen, $103 \mathrm{f}$.

${ }^{82}$ Grüttner, Armut, 29. 


\section{Frauen}

Bekanntlich war und blieb die SPD eine männerzentrierte Organisation. Dabei ist allerdings zu berücksichtigen, daß die Frauen vor allem in Preußen bis zum Reichsvereinsgesetz von 1908 formell gar nicht Mitglied einer Partei werden konnten. (Das Wahlrecht erhielten sie bekanntlich sogar erst 1919.)

Betrachtet man sich die unterschiedliche Vereinsgesetzgebung in den deutschen Ländern, so könnte man erwarten, daß Frauen in jenen Gegenden stärker vertreten waren, in denen schon vor 1908 eine großzügige, liberale Vereinsgesetzgebung bestanden hat, wie z.B. in Baden und Württemberg. Doch die Statistiken vermitteln ein anderes Bild: In Baden gab es kaum Frauen in der Partei ${ }^{83}$. Und in Württemberg waren im Jahre 1909 nur knapp 2\% der sozialdemokratischen Mitglieder Frauen, während der Reichsdurchschnitt immerhin damals schon bei etwa 10\% lag. Über die Hälfte der sozialdemokratischen Ortsvereine Württembergs wiesen 1910 überhaupt noch keine weiblichen Mitglieder auf, darunter befanden sich 12 Ortsvereine mit hundert und mehr männlichen Mitgliedern! Nur einige städtische Ortsvereine, unter ihnen die von Linken geführten Ortsorganisationen in Stuttgart, Göppingen und Esslingen, konnten bis 1914 den Anteil der Frauen an der Parteimitgliedschaft beträchtlich erhöhen ${ }^{84}$. Die weiblichen Mitgliederzahlen lagen in Göppingen sogar über dem Reichsdurchschnitt. Im Jahre 1911 zählte man dort 291 weibliche Mitglieder (im Jahre 1909 waren es erst 50!), d.h. die Frauen machten ungefähr ein Drittel der gesamten Mitgliederzahl Göppingens aus. Demgegenüber registrierte man im Jahre $1911 \mathrm{im}$ gesamten Deutschen Reich 107693 Frauen in der SPD, das waren 11\% der Gesamtmitgliedschaft $^{85}$.

Es fehlen noch vergleichende Studien, um die unterschiedlich große Repräsentanz der Frauen in den sozialdemokratischen Ortsvereinen ausreichend erklären zu können. Wer nach den bisherigen Ausführungen darauf spekuliert, daß der besonders hohe Anteil der Frauen in der Göppinger SPD als das Resultat des dort dominierenden linken Parteiflügels anzusehen sei, sieht sich schnell enttäuscht, wenn der Blick auf eine andere Parteiprovinz, nämlich auf die mehr und mehr reformistisch werdende Parteiprovinz Göttingen fällt. Denn auch im Göttinger Wahlkreis kletterte der Anteil der Frauen an der Parteimitgliedschaft bis zum Jahre 1913 auf immerhin $21 \%{ }^{86}$.

Nach ersten Überlegungen wirkten sich folgende zwei Faktoren für den Eintritt der Frauen in die SPD günstig aus: zum einen ein verhältnismäßig hoher Anteil von Frauen unter den Erwerbstätigen (vor allem im Dienstleistungsgewerbe), wie beispielsweise für Göttingen feststellbar ${ }^{87}$. Zum anderen zahlte sich vermutlich der Aufbau ei-

\footnotetext{
${ }^{83}$ Hierzu und zum Folgenden $S c h a d t / S c h m i e r e r$, Baden-Württemberg, $128 \mathrm{f}$. In Tübingen zählte man noch 1915 nur 6 weibliche Mitglieder in der dortigen SPD. Hans-Otto Binder/Gottfried Schmitt, „Bis alles bezahlt ist“. Aus den Protokollbüchern der Tübinger SPD 1894-1958 (Materialien zu einer Geschichte der Tübinger SPD 1, o. J. (1985)) 45.

${ }^{84}$ Wruerth, Göppingen, 48, 59.

${ }^{85}$ Errechnet aus: Fricke, Arbeiterbewegung, 245, 326.

${ }^{86}$ i: Saldern, Einwohner, 114.

${ }^{87}$ Vgl. auch Michels, Sozialdemokratie, 534.
} 
ner eigenen Frauengruppe (wie z.B. in Göppingen, Düsseldorf und in Göttingen) aus $^{88}$. Die eigene Frauengruppe erleichterte es wohl den Frauen, Kontakt zueinander zu finden; jedenfalls waren ihre Versammlungen offensichtlich gut besucht. Zudem gelang es diesen Frauengruppen, Themen zu diskutieren, die einen größeren Bezug zu ihrer eigenen Alltags- und Erfahrungswelt hatten, als dies bei den „normalen“ internen Parteiversammlungen der Fall war ${ }^{89}$.

Auch wenn solche Beobachtungen auf eine größere potentielle Aktivierbarkeit von Frauen für die Partei hindeuten als bisher angenommen, so dürfen keinesfalls die für eine politische Aktivierung ungünstigen sozio-kulturellen Strukturen, in denen Frauen lebten, vergessen werden (Stichworte: „Politik sei Männersache“, „Patriarchalismus“, „Doppelbelastung"). Mit Recht verweist beispielsweise Nolan darauf, daß die Frauen Fabrikarbeit meistens nur solange akzeptiert hātten, als sie nicht verheiratet waren bzw. keine Kinder hatten. Danach gingen sie nur dann noch in die Fabrik, wenn ihre Ehemānner zu wenig verdienten oder krank bzw. verstorben waren. War dies nicht der Fall, so nahmen sie Arbeit lieber mit nach Hause (Heimarbeit) oder übten Gelegenheitsarbeiten in der Nachbarschaft bzw. im Stadtquartier aus. Industriearbeit bedeutete ja bei den meist vorherrschenden patriarchalischen Grundstrukturen der Familie eine besonders hohe Doppelbelastung für die Frauen, wodurch der Eintritt in die Partei erschwert wurde. Der häufig dann spāter erfolgte Rückzug vieler (Ehe-)Frauen in die häuslich geprägte Alltagswelt war wohl auch - wenn er über viele Jahre anhielt - mit entsprechenden mentalen Verānderungen verbunden. Und die Arbeiterbewegung gab wenig gegenläufige Impulse. Die Arbeit der Ehefrauen (Haushaltsführung, Stundenarbeit bzw. „Zuverdienst“ sowie Kindererziehung) wurde nicht als eine mit der außerhäuslichen Lohnarbeit vergleichbaren Tätigkeit anerkannt und nicht als „öffentliche (politisierbare) Angelegenheit" angesehen; von daher verloren viele Frauen wohl auch ihren potentiellen Zugang zur Arbeiterbewegung. Im allgemeinen bestand für verheiratete Arbeitermütter nur dann eine Chance, in die Partei einzutreten, wenn die Ehemänner sie darin tatkräftig unterstützten. Es ist bekannt, daß es sich bei den „Hausfrauen“ in der SPD fast ausschließlich um Ehefrauen von Genossen handelte ${ }^{90}$.

${ }^{88}$ t: Saldern, Arbeiter-Reformismus, 174; Wuerth, Göppingen, 59. Darauf verweist auch Boll bei der Beantwortung der Frage, weswegen in Hannover wenige Frauen in der Partei organisiert waren. Boll, Massenbewegungen, 106.

${ }^{89}$ Dazu t: Saldern, Arbeiter-Reformismus, 174; vgl. auch Molly [Mary/ Nolan, Proletarischer Anti-Feminismus. Dargestellt am Beispiel der SPD-Ortsgruppe Düsseldorf, 1890 bis 1914, in: Frauen und Wissenschaft. Beitrāge zur Berliner Sommeruniversität für Frauen (Berlin 1976) $369 \mathrm{ff}$.

90 Michels, Sozialdemokratie, 534. Michels schātzte die Hausfrauengruppe als eine „nicht ganz geringe Minoritāt" ein; vgl. auch Richard J. Evans, Sozialdemokratie und Frauenemanzipation im Deutschen Kaiserreich (Berlin/Bonn 1979) 199 ff.; Willy Albrecht u. a., Frauenfrage und deutsche Sozialdemokratie vom Ende des 19. Jahrhunderts bis zum Beginn der zwanziger Jahre, in: Archiv für Sozialgeschichte XIX (1979) $472 \mathrm{f}$. Nach der Untersuchung von Niggemann waren die Frauen in der Partei meist jung und kamen aus proletarisch-handwerklichem Milieu. (Die sozialdemokratischen Führerinnen erkāmpften sich hāufig eine akademische Ausbildung.) Ungefähr die Hālfte (wenn nicht mehr) der weiblichen Mitglieder seien nicht erwerbstātig gewesen. Nigge- 


\section{Zusammenfassung:}

Die Frage, wer in die Partei ging, war in letzter Instanz zwar ein persönlicher Willensakt, der durch normale Quellenüberlieferungen nicht restlos aufgeklärt werden kann (zumal wenn die Möglichkeit von Oral History nicht mehr gegeben ist), doch gibt es zweifelsohne Determinanten, die die Wahrscheinlichkeit eines Parteieintritts erschwert oder erleichtert haben. Durch die Kombination von quantitativen und qualitativen Analyseschritten, wie sie in den vorliegenden Ausführungen versucht wurde, kommt man zu folgenden (vorläufigen) Ergebnissen:

1. Die Mitgliedschaft der SPD vor 1914 zeichnete sich durch eine relative Sozialeinheitlichkeit aus: Die SPD war - was die soziale Basis anbelangte - eindeutig eine Arbeiterpartei. Doch nur ein kleiner Teil der Arbeiterklasse fand den Weg in die Partei. Spezifische Erfahrungen und Realitātsdeutungen über Vergangenheit, Gegenwart und Zukunft mußten hinzukommen, um eine Entscheidung zu Gunsten eines Parteieintritts herbeizuführen.

2. Von besonderer Bedeutung für die Frage, wer den Weg in die Partei fand, war offensichtlich das mentale Rüstzeug beim Einstieg in die industriell-kapitalistische Welt. Hierbei kann die Bedeutung des Handwerks im Hinblick auf Ausbildungsangebote, Organisationen, Traditionen und Normen nicht überschätzt werden. Die handwerkliche Ausbildung verhinderte offensichtlich, daß der „Kultursprung“ sich zu abrupt vollzog und zur Überforderung der Betroffenen führte.

3. Wer sich der SPD anschloß, brach meist mehr oder weniger mit den Werten und Normen der traditionellen Lebenswelt. Er verband damit positive Zukunftshoffnungen, die aber nicht mehr auf der kleingewerblichen Selbständigkeit (als Meister), sondern auf der Existenz eines „lebenslānglichen“ Arbeitnehmers aufbauten. Der soziokulturelle Bruch mit der traditionellen Lebenswelt war am leichtesten dann zu vollziehen, wenn es auch keine objektiven Rückzugsmöglichkeiten mehr gab. (Arbeiterbauern und Frauen hatten beispielsweise oft immer noch die Möglichkeit bzw. die Hoffnung, eine andere Existenz als die eines/einer Industriearbeiters/arbeiterin aufzubauen, sei es durch die eigenständige Bewirtschaftung von Grund und Boden, sei es durch Ehe und Familie. Sie waren deshalb für die Partei schwer zu gewinnen.)

Allerdings mußte der Bruch mit der traditionellen Lebenswelt auch mental zu bewältigen sein, was beispielsweise bei den nach Hamborn gezogenen Arbeitern offensichtlich nicht möglich war. Dagegen konnte es bei einem "gestuften“ sozio-kulturellen Sprung zu einer relativ „positiven“ Einstellung gegenüber der kapitalistisch-industriellen Entwicklung und der bürgerlichen Gesellschaft kommen, insofern diese als ein notwendiges Durchgangsstadium für eine bessere, d.h. sozialistische Zukunft betrachtet wurde. (Der Bruch mit der bürgerlichen Gesellschaft hatte also eine ganz andere Struktur als der Bruch mit der traditionellen Lebenswelt.) Eine solche Zukunfts- 
deutung setzte allerdings meist ein gewisses Niveau an Ausbildung und einen akzeptablen Arbeitsplatz (möglichst in einem Leitsektor wie in der metallverarbeitenden Branche) voraus. Es ist vielleicht auch deswegen kein Zufall, wenn vor allem jene sich der Partei anschlossen, die auf dem Höhepunkt ihres individuellen Lebenszyklus standen: Individueller und kollektiver Fortschrittsglaube waren dann ineinander verzahnt.

4. Die Bedeutung der Gewerkschaften als Politisierungsinstanz muß hoch veranschlagt werden. Der Eintritt in eine Gewerkschaft führte teilweise (längst nicht immer) auch zu einer Mitgliedschaft in der SPD. Dahinter stand wohl die Erkenntnis, daß die Verfolgung ökonomischer Interessen (durch die Gewerkschaften) eine Verfolgung politischer Interessen (durch die Partei) dringend bedürfe.

5. Die SPD tat sich schließlich bei der Agitation in Parteizentren relativ leicht, wenn die kulturelle bürgerliche Hegemonie durch die Arbeiterbewegung aufgebrochen werden konnte und urbane Dichte mit gewachsenem Arbeitersozialmilieu zusammenfiel. In den Parteiprovinzen, in denen die kulturelle bürgerliche Hegemonie nahezu allumfassend erschien, wollten viele potentielle sozialdemokratische Mitglieder anonym bleiben; deshalb gaben sie lieber der SPD im geheimen - nämlich bei den Reichstagswahlen - ihre Stimme. Wer sich in der Parteiprovinz doch schließlich dazu durchrang, in die SPD einzutreten, ließ es häufig nicht zu einem völligen Bruch mit der traditionalen Welt kommen, sondern lebte weiterhin in jenen Spannungen, die durch die Ungleichzeitigkeit des Entwicklungsablaufs erzeugt wurden.

\section{Nachwort:}

Als Bausteine zur Konstruktion eines sozialdemokratischen Idealtypus um die Jahrhundertwende können gelten:

Lohnabhāngig, mānnlichen Geschlechts, evangelisch oder aus der Kirche ausgetreten, Volksschulbildung, handwerkliche Ausbildung, in der metallverarbeitenden Branche oder im Bau- und Holzsektor (vor allem in Mittelbetrieben) tätig; gewerkschaftlich organisiert, zwischen 20 und 35 Jahre alt, verheiratet; in einem urbanen Parteizentrum lebend. 
